Research Article

\title{
Q-CAM: Queue Monitoring System using Camera
}

\author{
Farah Nadiah Mohammad Ramlee, Radzi Ambar*, Mohd Helmy Abd Wahab, Chew \\ Chang Choon and Muhammad Mahadi Abd Jamil \\ Universiti Tun Hussein Onn Malaysia, Johor, Malaysia \\ farahnadiah_mr@yahoo.com; helmy@uthm.edu.my; ccchew@uthm.edu.my; mahadi@uthm.edu.my \\ *Correspondence: aradzi@uthm.edu.my
}

Received: 17th October 2020; Accepted: 20th December 2020; Published: 20 ${ }^{\text {th }}$ March 2021

\begin{abstract}
Development and design of mobile queue counter monitoring system is an alternative way for people who visit service premises to monitor queue number through phone without having to be at the place to wait for their turn. A long waiting line and waiting time are the reasons why this project had been developed. Many related systems had been introduced by different companies but due the high cost of installation and maintenance, some service companies had difficulties to implement the system. However, the proposed mobile queue counter monitoring system called Q-CAM is an add-on device which will be installed with the existing system and uses only ESP32 camera and FTDI Programmer as hardware development. A smartphone application is developed by using MIT App Inventor to ease the customer to monitor queue number on the app. In this work, the hardware designs of the propose device is described. Furthermore, the steps to develop the smartphone app is demonstrated. Preliminary experimental results show that the average time required to display a captured queue number image on the app were 5 seconds (during strong Wi-Fi signal) and 10.8 seconds (during weak Wi-Fi signal).
\end{abstract}

Keywords: Queue monitoring system; ESP32 camera; smartphone application; Wi-Fi; QMS

\section{Introduction}

Queuing system in a customer service can be described as a system where the arrival of customers is the input and the served customer as the output. Usually in this system, the aim is to control customer flow and the customer has to wait for their turn to get the service. There are various queue systems currently implemented in Malaysia that had been implemented at most establishment such as in finance, government and healthcare sectors that manage customer flow and queues ${ }^{1}$. This system had been used since decades ago and became the main queue system utilized in Malaysia. These systems are necessary to ensure disciplined and efficient guided-customer service.

Basically, queuing is a line of people or things waiting for their turn to be handled normally in a first-come-first-handled order. However, problem arises when queue become longer than anticipated and increase wait times which leads to the prime reason of customer dissatisfaction ${ }^{2}$. Customer services-oriented companies have been encountering difficulties of long line queue. Premises tend to get crowded especially during peak periods and also can cause some queuing problems such as balking and reneging. Balking means customers that refuse to join the queue for services, while reneging is when the customer decided to leave the queue after waiting too long for

${ }_{1}$ https://blog.wavetec.com/en/queue-management-system-in-malaysia

${ }^{2}$ https://www.thestar.com.my/news/nation/2019/02/28/apad-investigating-video-of-man-losing-his-cool-says-he-hadntwaited-that-long/

Farah Nadiah Mohammad Ramlee, Radzi Ambar, Mohd Helmy Abd Wahab, Chew Chang Choon and Muhammad Mahadi Abd Jamil, "Q-CAM: Queue Monitoring System using Camera", Annals of Emerging Technologies in Computing (AETiC), Print ISSN: 2516-0281, Online ISSN: 2516-029X, pp. 137-145, Vol. 5, No. 5, $20^{\text {th }}$ March 2021, Published by International Association of Educators and Researchers (IAER), DOI: 10.33166/AETiC.2021.05.017, Available: http://aetic.theiaer.org/archive/v5/v5n5/p17.html. 
the services ${ }^{3}$. Therefore, a queue management system (QMS) must handle and organizes queue arrangement effectively.

Conventional ways to handle queues such as distributing paper tickets printed with queue number lead to several issues such as littering paper tickets and long queues or wait times at premises. Alternative ways to solve this problematic customers' queuing experiences are by implementing e-services or mobile QMS. A work done in [1] proposed a QMS which can generate and send queue number via SMS. Lin et al. [2] proposed a smartphone app based QMS that displays pops up notification messages to customer using Bluetooth beacon technology. A study in [3] proposed a smartphone app based QMS that enable restaurant customers to view, book and manage his/her queue prior arrival to the restaurant. There are also studies such as [4] and [5] that propose queue waiting time estimation based on numerical simulation to minimize waiting time. A QMS has been implemented in a hospital that can display real-time queue data and also room availability via smartphone and website ${ }^{4}$. Based on the above, mobile QMSs are becoming an interesting research area where new devices and mobile applications are introduced. However, premise owners tend to use existing queue counter display that they have invested in their premises. There are QMSs that are commercially available, where real-time images from cameras are used to update queue number, expected wait time and time of entry that can be monitored via mobile device ${ }^{5,6}$. However, the systems are costly and involve complex implementation.

Thus, this paper describes the design and development of a mobile queue counter monitoring system using camera called Q-CAM. The objectives of the work are two-fold; to provide an alternative way to reduce waiting time at premises using smartphone, and also reduce mobile QMS implementation costs where the system serves as an add-on device for existing queue counter display in premises. This paper is organized as follows. Section 2 describes the detail design of the proposed Q-CAM including hardware and software components. Section 3 introduces the design of the system. Then, section 4 discussed the experiments to show the usefulness and performance of Q-CAM in achieving the objectives, followed by a brief conclusion and future recommendation in section 5 .

\section{Proposed Method}

This section describes the project overview, project design, methods, hardware and software requirement that will be used in this project. Figure 1 shows the block diagram of the proposed mobile ${ }^{2}$ queue counter monitoring system. This project is an add-on device for any existing queue counter devices used in premises. The input of the device is the captured images of the turn number displayed on the queue counter. As shown in the figure, the hardware parts utilized in the device are an ESP32 camera and an FTDI (Future Technology Devices Interglobal) Programmer. The camera captures images that are transmitted to an Android-based smartphone application (app) built specially for the queue counter monitoring system. In this app, user is able to monitor and see the current queue number at the premise by the image captured from the camera from remote location.

\subsection{Hardware Components}

As shown in Figure 1, an ESP32 camera module is used in this project to capture the turn numbers from queue counter display. The ESP32 camera is a small-sized module that can operate independently as a minimum system with a footprint of only $27 \mathrm{~mm} \times 40.5 \mathrm{~mm} \times 4.5 \mathrm{~mm}$ and a deep sleep current of up to 6Ma. It consists of two parts which are the main board that is an ESP32 module mounted on a larger board and a separate camera module. A flex cable from the camera slots into the main board that is based on an ESP32 module. The module is a programmable mic rocontroller with built-in Wi-Fi and Bluetooth, with an additional 4MB of external RAM.

\footnotetext{
${ }^{3}$ https://www.americaninno.com/boston/jockeying-faffing-balking-the-science-behind-waiting-in-line-from-mit/

4 https://hampg.moh.gov.my/qms/\#!/

${ }^{5}$ https://www.retailsensing.com/queue-management.html
} 
The board is equipped with a memory card slot and a camera connector that can be connected to an OV2640 camera module. However, the memory card is not used in this project development. In this work, once a new image is captured, the previous captured image will be deleted.

Since this is a Wi-Fi enabled camera, IP address is required in order to observe the captured images on camera control page. Figure 2 shows the IP address printed on the computer's serial monitor after the source code was uploaded into ESP32 camera module. Then, the IP address can be typed on a web browser to access camera streaming server on local network. These steps are necessary to operate the ESP32 camera module. Table 1 shows ESP32 camera specifications.

Figure 1 also shows a FTDI programmer that is used in this project. The FTDI programmer works for converting TTL (Transistor-Transistor Logic) serial transmission to USB signals, where it is used to upload the source codes from personal computer (PC) to ESP32 board via the U0RXD and U0TXD serial pins. This is done due the fact that there is no USB port on ESP32 board to be connected to the PC. This adapter support both $3.3 \mathrm{~V}$ and $5 \mathrm{~V}$ operations.

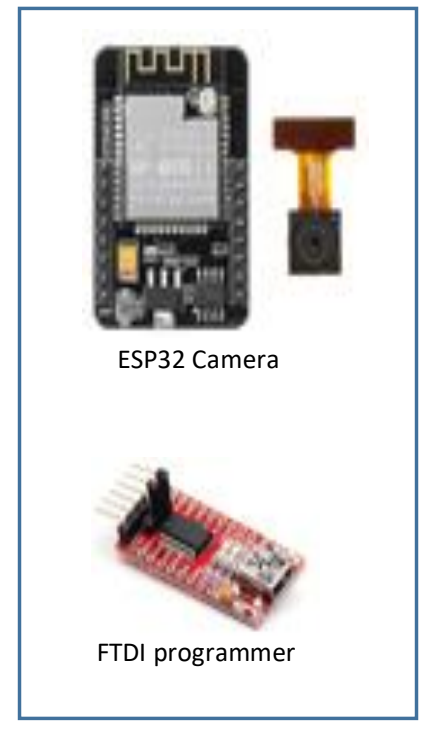

Hardware

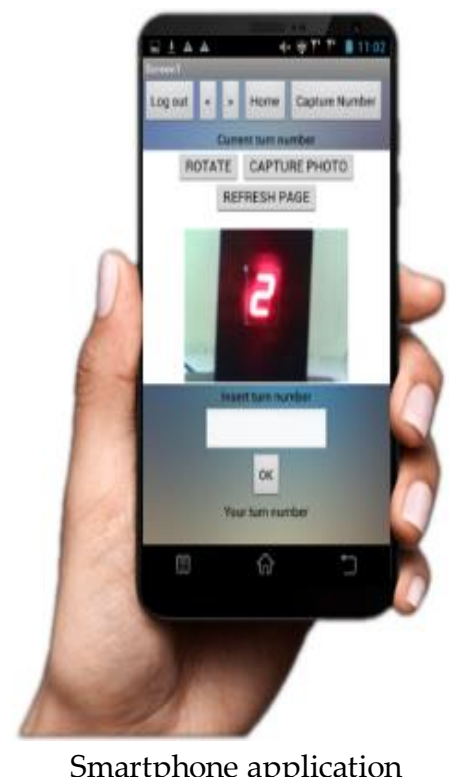

Smartphone application

Figure 1. Overview of the proposed queue monitoring system

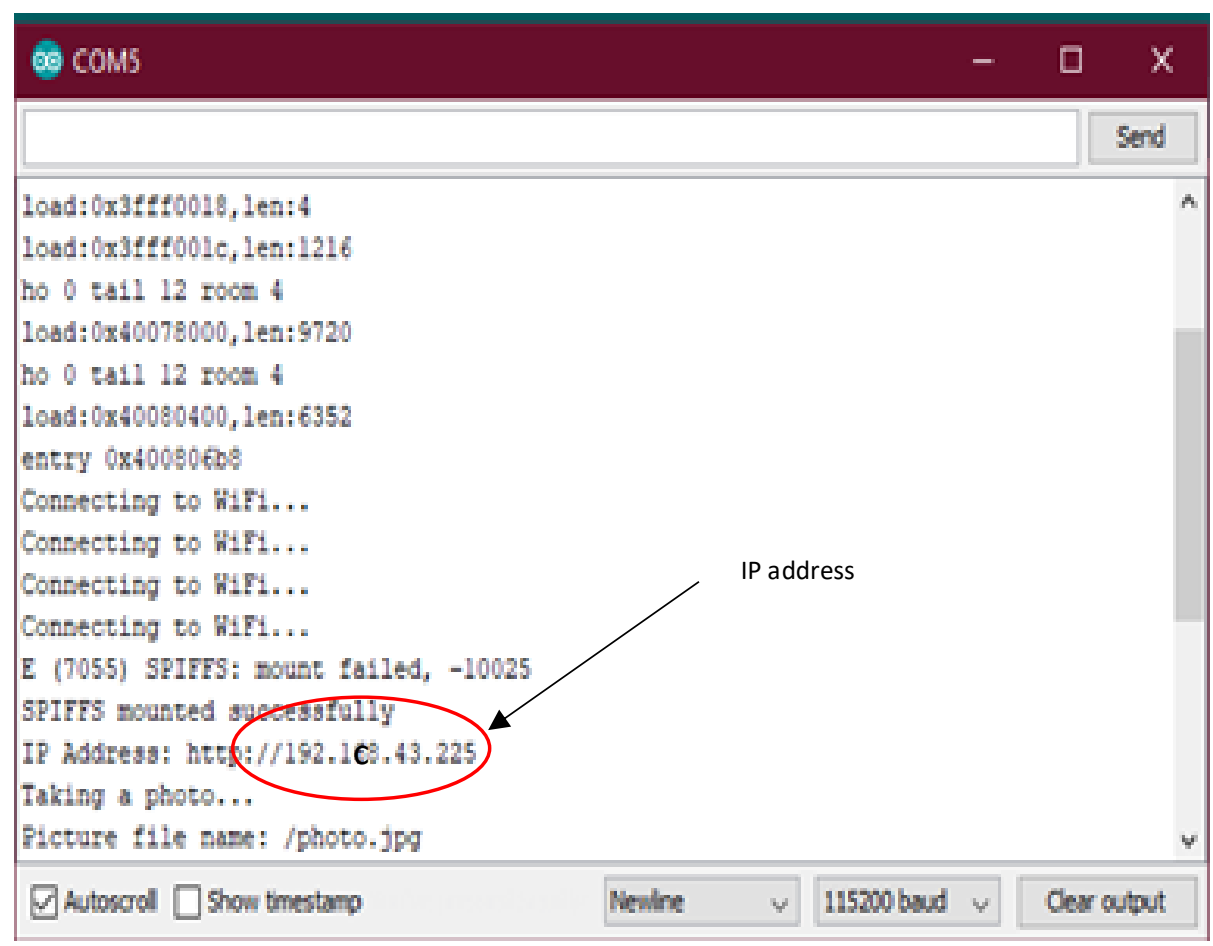

Figure 2. IP address displayed in serial monitor 
Table 1. ESP32 Camera Specifications

\begin{tabular}{|l|l|}
\hline Module model & ESP32-CAM \\
\hline Package & DIP-16 \\
\hline Size & $27^{*} 40.5^{*} 4.5$ \\
\hline SPI flash & Default 32 Mbit \\
\hline RAM & $520 K B$ SRAM + 4M PSRAM \\
\hline Bluetooth & Bluetooth 4.2 BR/EDR and BLE standard \\
\hline Wi-Fi & $802.11 \mathrm{~b} / \mathrm{g} / \mathrm{n}$ \\
\hline Support interface & UART, SPI, I2C, PWM \\
\hline Support TF card & Maximum support 4G \\
\hline IO port & 9 \\
\hline UART baudrate & Default 112500 bps \\
\hline Image output format & JPEG (OV2640 support only), BPM, Grayscale \\
\hline
\end{tabular}

\subsection{Software Requirements}

Arduino integrated development program (IDE) is used to program the source codes for the ESP32 board. Therefore, the IDE should be installed in the ESP32 board prior to be used. The ESP-32 board is uploaded with the source code that can sends commands to capture image and send the image to a smartphone application via Wi-Fi. This work uses a web application development environment called MIT App Inventor to create application software (app) for smartphones and tablets. This app inventor can only develop application for Android phone using a web browser and either a connected phone or emulator.

\section{Design of the System}

\subsection{Circuit Design}

Figure 3(a) shows an illustration of the circuit connection between ESP32 camera module and FTDI programmer. As shown in the figure, the circuit is simple, where the ESP32 camera is connected with FTDI programmer via four wire connections as this camera module does not come with USB connector. The FTDI programmer works for converting TTL serial transmission to USB signals, and also to upload source code from a PC through the UOR and UOT pins (serial pins). Figure 3(b) shows the experimental setup to test ESP-32 Cam with FTDI programmer. The figure shows the connection of the ESP32 camera module with the FTDI programmer. Detail of the experiment is discussed in the next section.

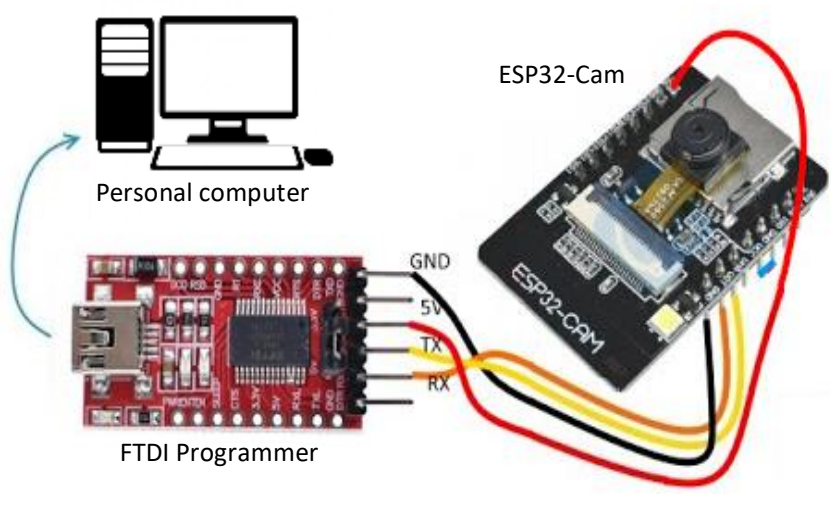

(a)

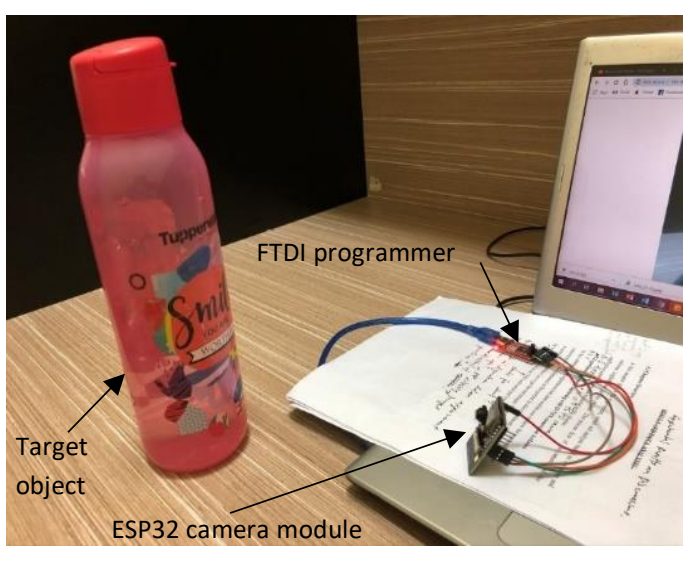

(b)

Figure 3. (a) Circuit connection for ESP32 camera module with FTDI programmer, (b) the actual experimental setup to test ESP32 camera

\subsection{Android-based Smartphone Application Design}

As described in the previous section, the Android-based smartphone app is developed using MIT Apps Inventor on a personal computer. Basically, there are two ways to test the app developed 
using the MIT Apps Inventor; (a) using Android Package (APK) file of the developed app and saved it in ZIP format to be downloaded directly to Android smartphone, or (b) scanning the QR code obtained from MIT App inventor. In this work, method (b) was selected because the developed app can be tested in real-time on any Android-based smartphone. However, another app called MIT AI2 Companion app needs to be downloaded in an Android smartphone beforehand to scan the QR code from MIT Apps Inventor that can automatically download and test the developed app.

Based on several tests, it is found that a strong Wi-Fi connection is crucial in order to send the captured image from ESP32 camera module to the developed app. Furthermore, the method of scanning the QR code to download and test the developed the app has a drawback where the QR code is valid to be used within 2 hours only, and user is required to get new QR code after 2 hours. However, this is enough for a short period of testing purposes.

Figure 4 shows the developed Android-based smartphone app for queue monitoring system. Figure 4(a) shows the condition when the app receives the captured image successfully. Figure 4(b) shows the condition when the apps is unsuccessful to receive image due to low connection of Wi-Fi.

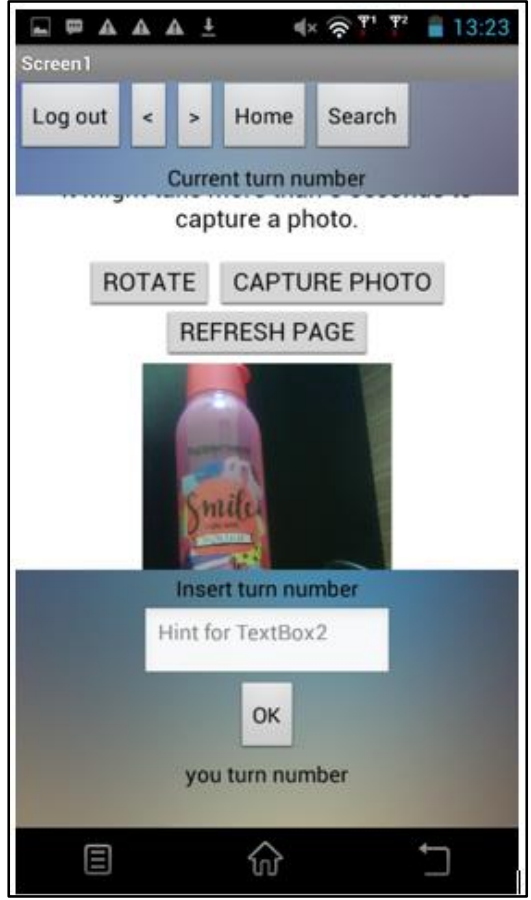

(a)

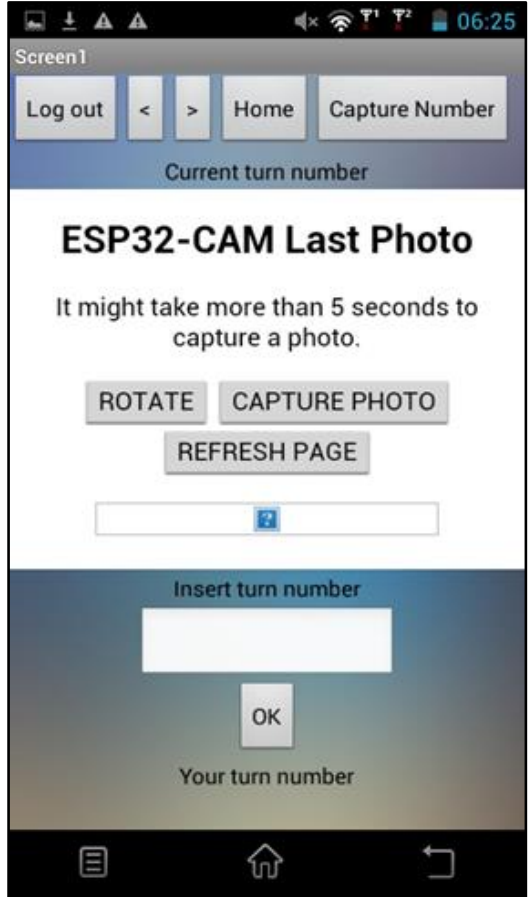

(b)

Figure 4. The developed smartphone app. (a) Image received successfully, (b) Unsuccessful in receiving image

As shown in Figure 4(a), the single app page consists of three parts. On the top half of the app, there are Log out, Home, Search and scroll (left and right) buttons. The Log out button is to exit from the app. Home button is to refresh the page. As the app is in preliminary test version, the scroll and search buttons are not used. At the center of the page shows the internet web browser that displays the captured image taken from the ESP32 camera. The web browser is opened based on the IP address from ESP32 camera. As shown in Figure 4(b), the web browser displays ROTATE, CAPTURE PHOTO and REFRESH PAGE buttons. When CAPTURE PHOTO button is pushed, it will instruct the ESP32 camera to capture an image to be sent to the app. REFRESH PAGE button is to refresh the page if the capture image is not displayed. Currently, the ROTATE function is not being used. On the lower half of the page shows a text box to type customer's turn number. OK button is clicked to save the turn number in the app so that the customer can easily keep reference of turn number.

\section{Experimental Steps and Results}

There are two experiments that have been carried out in this work. The first experiment is to verify the usefulness of the ESP32 camera in capturing a target object. The second experiment is to 
show the performance of the developed app to display the captured image from ESP32 camera. The detail of each experiment is described in the next subsections.

\subsection{Experiment on ESP32 Camera}

Figure 3(b) shows the experimental setup for verifying the usefulness of ESP32 camera. As shown in the figure, the ESP32 camera is connected with FTDI programmer. The ESP32 camera is positioned in front of a target object which is a water bottle.

Experimental Steps. First, ESP32 camera is connected to FTDI programmer. 5V power is used supply to power FTDI programmer. Then, source code is uploaded to FTDI programmer. After the code uploaded successfully, serial monitor is opened to get the IP address. The IP address is copied and pasted on the internet browser. The camera is placed in static position in front of the object to capture a good image. Then, the camera starts to capture image after clicking CAPTURE PHOTO button. Finally, the test image can be observed through internet browser.

Experimental Results. As ESP32 Cam is a Wi-Fi-enabled camera, obtaining IP address after uploading the code is an important step prior to view the test image. Then, the camera server can be access on local network by inserting the IP address on the internet browser to view the captured image. Figure 5(a) shows how the IP address for the ESP32 is obtained after the code is uploaded into the ESP3 board. The IP address should be displayed on the serial monitor on the condition that the camera received a strong Wi-Fi connectivity. Otherwise, it would not have displayed the IP address on serial monitor as shown in Figure 5(b).

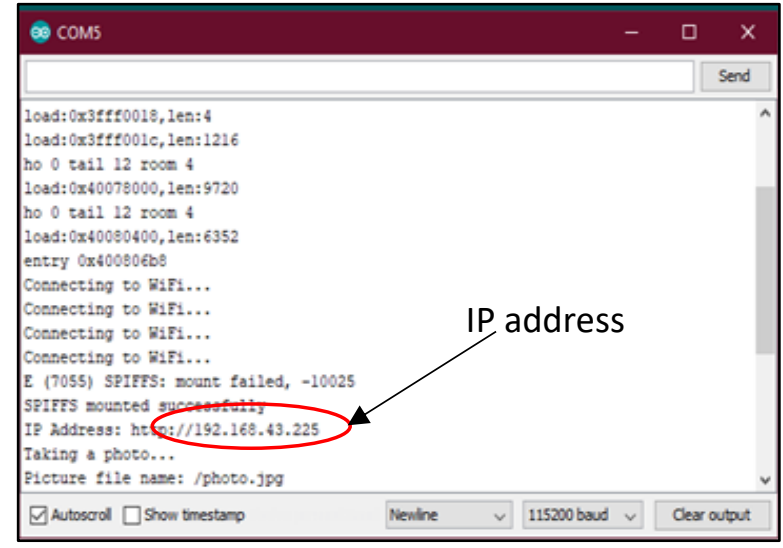

(a)

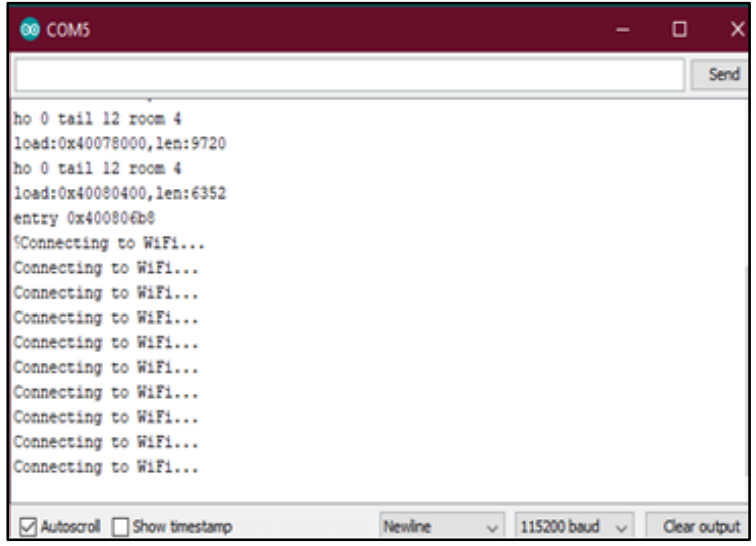

(b)

Figure 5. (a) Getting the IP address after downloading the code. (b) No IP address displayed due to weak WiFi connection

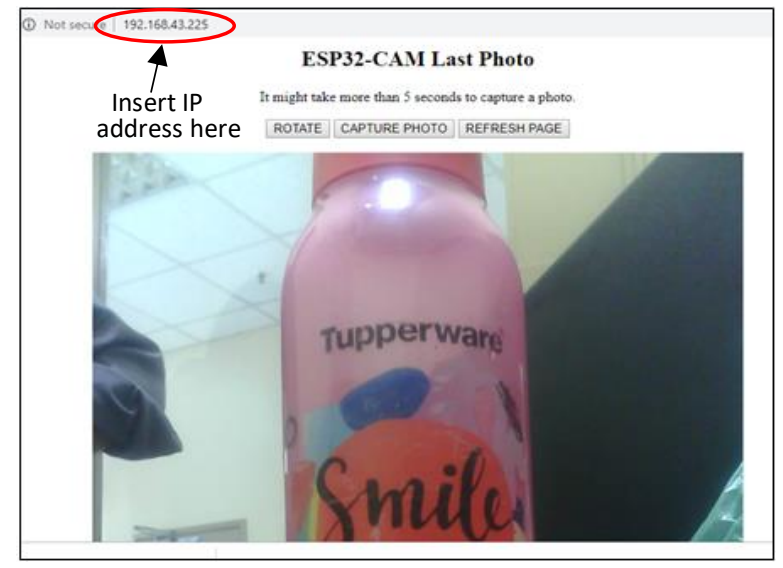

(a)

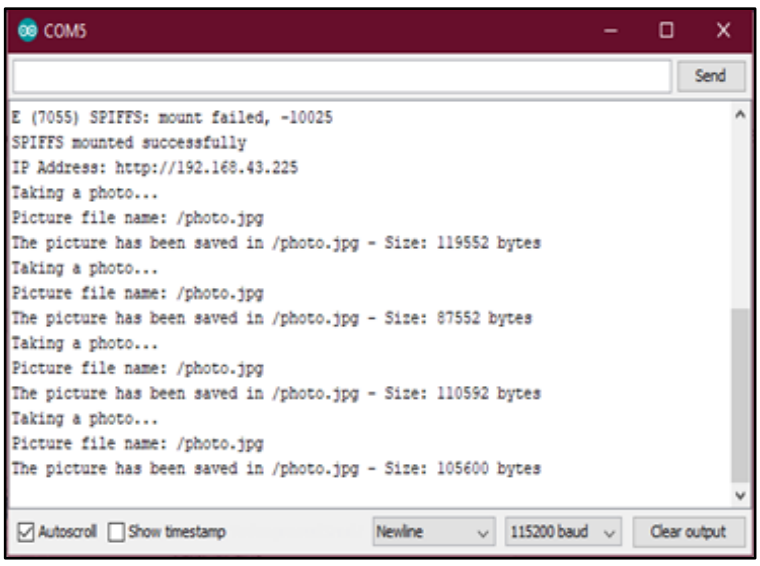

(b)

Figure 6. (a) Test image. (b) Serial monitor showing the captured images are saved

Next, by inserting the IP address on the internet browser, the test image can be viewed immediately after capturing a picture as shown in Figure 6(a). The average time taken to display a captured image during this experiment was approximately 5 seconds. The quality of the image taken 
using this camera is much better compared to OV7670 camera module that we have tested. The ESP23 camera produces coloured and clear image. Just by clicking on CAPTURE PHOTO button, new image will be captured and saved as shown in serial terminal in Figure 6(b).

Discussion. In this experiment, ESP32 camera functioned well as it produced a better quality image in short time. As described previously, low-cost camera such as OV7670 module only produces monochrome images. However, ESP32 camera is able to produce coloured images. Furthermore, it has been observed that the procedures to capture images are simpler and more convenient which is suitable to be applied in this project. However, the downside of this camera module is, it requires a strong Wi-Fi connection to smoothen the camera operation. As a solution, for experimental purposes, the camera will be connected to personal hotspot device to make the whole system works perfectly.

\subsection{Queue Counter Monitoring System Experiment}

A full test operation of the proposed queue counter monitoring system have been carried out to verify the performance of the system. Figure 7 shows the experimental setup. The ESP32 camera and FTDI programmer are packaged in the small black box with an opening for the camera to capture images. In front of the camera is a mock-up single number queue counter. A button is located below the queue counter to change the turn number displayed using a 7-segment display.

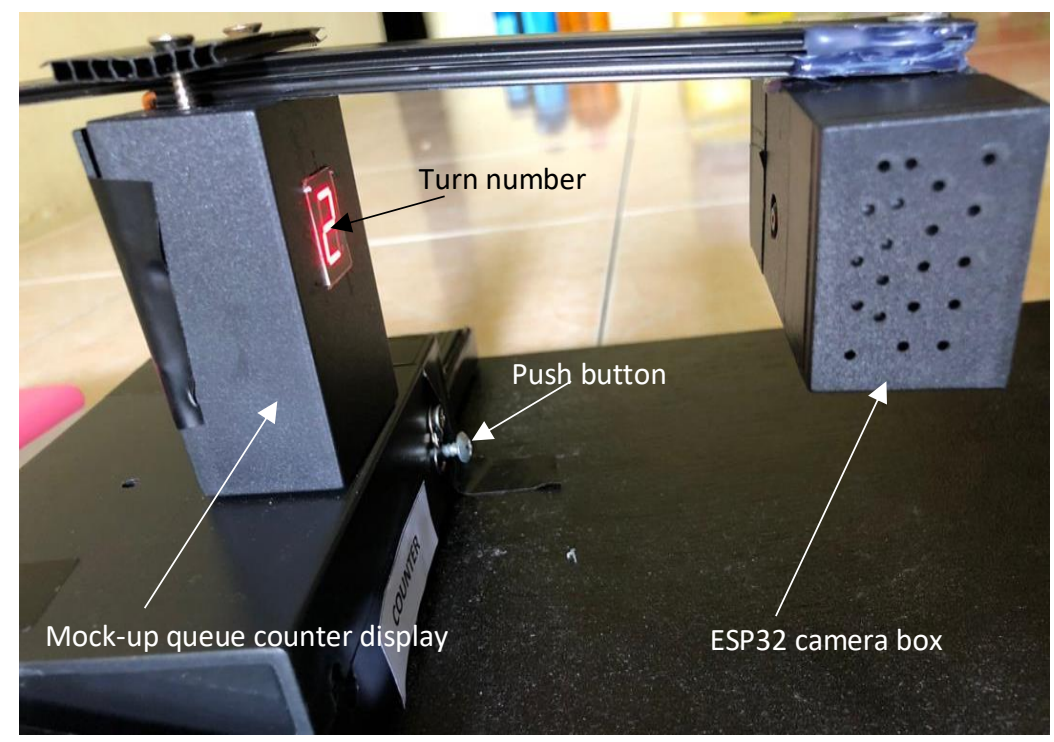

Figure 7. Serial monitor showing the captured images are saved

Experimental Steps. First, the queue counter and ESP32 camera is turned ON. After start up the developed smartphone app, "Capture number" button is pushed to access ESP32 camera server. Click CAPTURE PHOTO button to capture image. Then, click REFRESH PAGE button to display the captured image on the app. Push the push button on the queue counter to change the turn number. Then, repeat step 4 to 6 to display the captured image on the app.

Experimental Results. In this experiment, the 7-segment can display numbers from 0 to 9 to represent the turn number for a queue counter in a real situation. This 7 -segment is controlled manually by pressing the push button as shown in Figure 7. In a real situation, the user of the app can use obtain the current turn number from remote location. However, the user must download the developed app. Figures 8 shows the captured image which display different turn number from the 7segment display.

Discussion. After experimenting with the system, it is found that strong Wi-Fi connection plays an important role in order to operate the system perfectly. This experiment have also tested the effect of low and high Wi-Fi connection towards the performance of transmitting the captured image from ESP32 camera to the smartphone app. The time taken to capture and display captured images from ESP32 camera to smartphone app were observed and recorded. The speed of the Wi-Fi connection is represented by signal bar on Wi-Fi signal indicator on the smartphone as shown in Figure 9. 

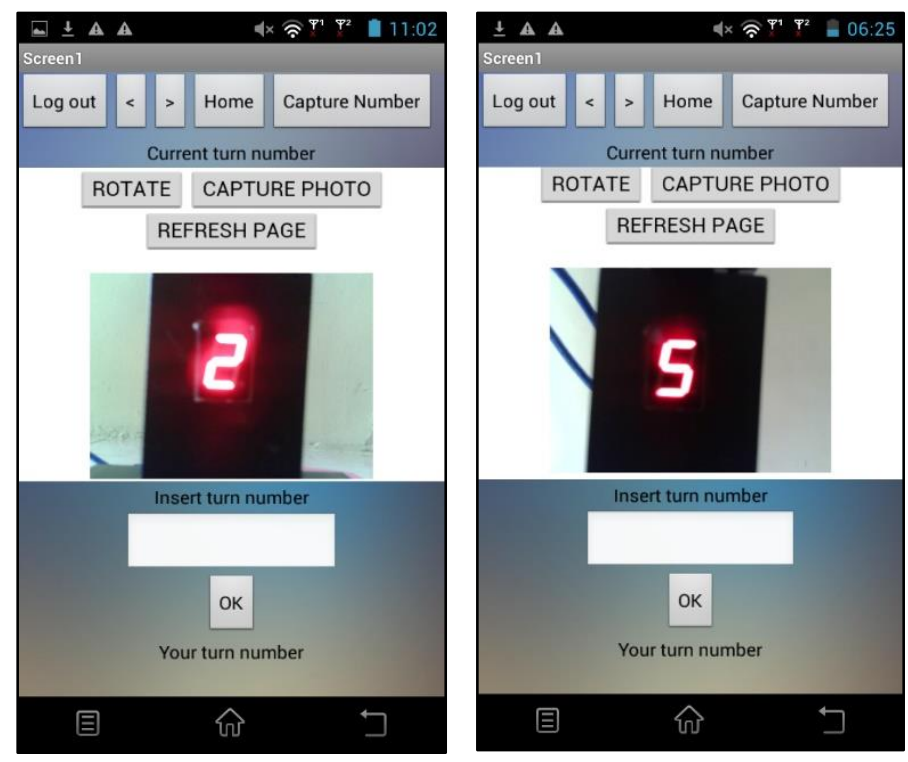

Figure 8. The app displays turn number 2 and 5

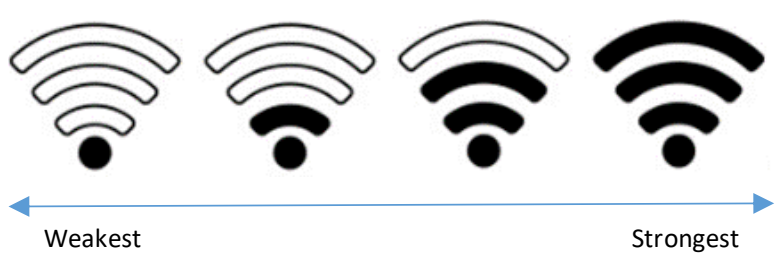

Figure 9. Wi-Fi signal strength indicator

First, both camera and smartphone were connected to low Wi-Fi connection. A stop watch was used to record the time taken from the step 1 in the experiment steps above until the turn number image is displayed on the developed apps. First, experimental steps 1 to 4 in the experiment steps above were carried out, then, if no image displayed, the CAPTURE PHOTO button was clicked concurrently until the turn number image was displayed and the time taken was recorded. The same experiment steps were carried out high Wi-Fi connection. The steps above were repeated for each turn number from 0 to 9 for each low and high Wi-Fi connection to observe the camera performance.

Tables 2 shows the comparison of the time taken to display the queue number images during weak and strong Wi-Fi signals respectively. From the experiment, the average time required to display the captured images when both camera and smartphone were connected to weak signal strength was 10.8 seconds. However, it took an average time of 5 seconds to display the captured images on the app when Wi-Fi signal was strong. There are few possibilities on why it took longer time to display images when the camera is connected to weak Wi-Fi connection, and device interference is one of the causes. Sometimes intermittent signal can occur caused by interference from other devices hence it can disturb Wi-Fi signal strength. Moreover, one of the most common causes of weak Wi-Fi connection is distance between the ESP32 camera and smartphone. It is found that since the camera is connected to a personal hotspot device, the mobile device should be placed closer to the receiver to obtain stronger signal.

Table 2. Comparison of the time taken to display queue number images on the app during weak and strong Wi-Fi signals

\begin{tabular}{|c|c|c|}
\hline \multirow{2}{*}{ Queue Number } & \multicolumn{2}{|c|}{ Time Taken (s) } \\
\cline { 2 - 3 } & During weak Wi-Fi signal & During strong Wi-Fi signal \\
\hline 0 & 13 & 7 \\
\hline 1 & 12 & 4 \\
\hline 2 & 12 & 4 \\
\hline 3 & 11 & 5 \\
\hline 4 & 10 & 5 \\
\hline 5 & 10 & 5 \\
\hline 6 & 9 & 4 \\
\hline
\end{tabular}




\begin{tabular}{|l|l|l|}
\hline 7 & 10 & 6 \\
\hline 8 & 11 & 5 \\
\hline 9 & 10 & 6 \\
\hline
\end{tabular}

\section{Conclusion}

Mobile queue counter monitoring system is useful for customer in premises such as post office, clinics and banks where the user can monitor their turn number without having to be at the premise for hours while waiting for their turn. In this work, a low-cost mobile queue counter monitoring using ESP32 camera called Q-CAM have been developed successfully. Q-CAM is able to capture images and the smartphone app can display the captured images. The results from the experiments show that the system operated effectively and achieved the objectives. It is also found that strong Wi-Fi connection is required for prompt display of image on app. Future work may consists of adding multiple image displays in the app, in a situation where multiple queue counter exists in premise. Further upgrades and experiments will be carried out to improve the performance of the system.

\section{Acknowledgement}

The authors would like to thank the Research Management Center (RMC), UTHM and Ministry of Higher Education for sponsoring the research under Tier 1 Research Grants (H161).

\section{References}

[1] Aiman ZJ, Norfadzlia MY, Tole S (2016) Arduino Based Paperless Queue Management System, Telkomnika, vol. 14, no. 3, pp. 839-845, doi: http://dx.doi.org/10.12928/telkomnika.v14i3.3114.

[2] Lin C, Jhu G and Siao S (2019) An Innovative Queue Management System Based on Bluetooth Beacon Technology. 2019 IEEE Int. Conf. on Comp. Sci. \& Eng. (CSE) and IEEE Int. Conf. on Embedded and Ubiquitous Computing (EUC), New York, NY, USA, 2019, pp. 427-430, doi: 10.1109/CSE/EUC.2019.00085.

[3] Ramasamy R, Haw S, Chua F (2018) Casual dining restaurant queue management system to optimize decision making in table seating arrangement. Knowledge Management Int. Conf. (KMICe), pp. 18-22, Available: http://www.kmice.cms.net.my/ProcKMICe/KMICe2018/pdf/CR8.pdf.

[4] Patel JJ, Chaudhary RM, Patel PA, Makwana P (2012) Minimize the Waiting Time of Customer and Gain more Profit in Restaurant using Queuing Model. Int. J. of Eng. Res. and Appli. (IJERA) 2(3): p.1422-1425, Available: http://www.ijera.com/papers/Vol2_issue3/IL2314221425.pdf.

[5] Hermanto RPS, Suharjito, Diana, Nugroho A (2018) Waiting-Time Estimation in Bank Customer Queues using RPROP Neural Networks. Procedia Computer Science, vol. 135, pp. 35-42, doi: https://doi.org/10.1016/j.procs.2018.08.147. 